\title{
In Memory of Eberhard Zeidler
}

\author{
Jürgen Jost ${ }^{1} \cdot$ Hoang Xuan Phu ${ }^{2}$
}

Published online: 2 January 2019

(C) Vietnam Academy of Science and Technology (VAST) and Springer Nature Singapore Pte Ltd. 2019

Eberhard Zeidler (Fig. 1), our friend, colleague and mentor, passed away on November 18, 2016, after a long illness, and this issue of the Vietnam Journal of Mathematics is dedicated to his memory.

Eberhard Zeidler was born in Leipzig on October 6, 1940. Leipzig remained his hometown throughout his life. Here, he grew up, attended school, and studied mathematics at the university. Even though he was relegated from the university for three years for political reasons and because of his upright personality, he could already receive his $\mathrm{PhD}$ degree in 1967 with Herbert Beckert, with his dissertation "Über eine Klasse nichtlinearer singulärer Randwertaufgaben der Funktionentheorie mit Symmetrieverhalten" (On a class of nonlinear singular boundary value problems in function theory with symmetry). In 1970, he qualified as a professor, with the habilitation thesis "Zur Theorie und Praxis einer Klasse freier Randwertprobleme der ebenen Hydrodynamik" (On the theory and praxis of a class of free boundary value problems of planar hydrodynamics).

He went on to found the field of nonlinear functional analysis with his impressive books, first in German and later in an expanded 5 volume English edition "Nonlinear Functional Analysis and its Applications" [1-5]. From 1974 to 1996, Eberhard Zeidler was Full Professor for Analysis at the University of Leipzig. When the wall that separated East from West Germany came down in 1989, together with two colleagues, he took the initiative for restructuring the University of Leipzig. In 1996, he could found the new Max Planck Institute for Mathematics in the Sciences in Leipzig, together with Jürgen Jost and Stefan Müller. He could then devote his enormous scientific energy to the main theme of his life, a grand vision of the unity of mathematics and physics. In this direction, he produced several series of impressive and encompassing monographs. The crowning achievement, a planned 6 volume treatise on "Quantum Field Theory," however, has remained unfinished because

Jürgen Jost

jost@mis.mpg.de

Hoang Xuan Phu

hxphu@math.ac.vn

1 Max Planck Institute for Mathematics in the Sciences, Inselstraße 22, 04103 Leipzig, Germany

2 Institute of Mathematics, Vietnam Academy of Science and Technology, 18 Hoang Quoc Viet Road, Cau Giay District, Hanoi, Vietnam 


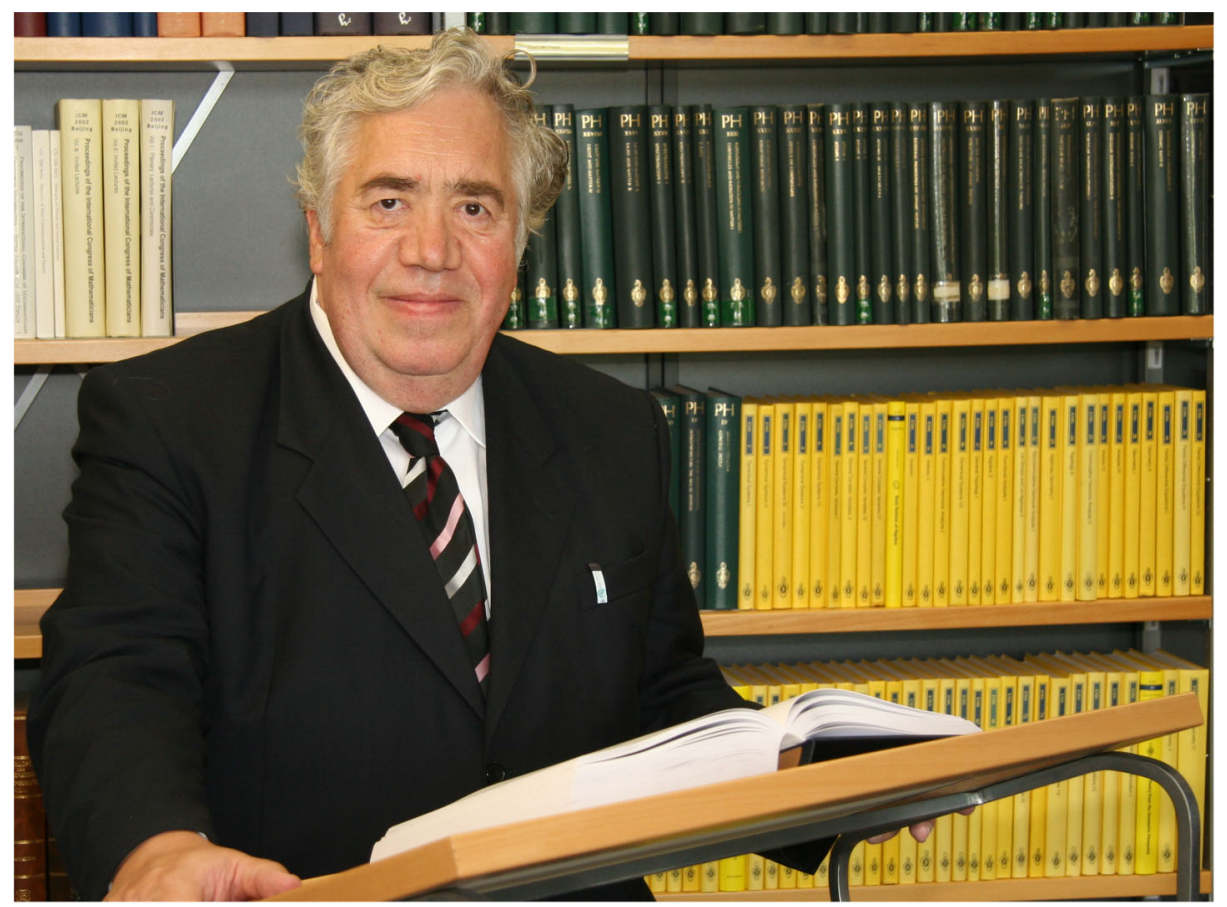

Fig. 1 Eberhard Zeidler 1940-2016

of his untimely death. Three of the six volumes have appeared [6-8], however, and currently Jürgen Tolksdorf is working on completing the fourth on the basis of his manuscripts.

He educated a substantial number of doctoral students, several of whom had successful academic careers themselves, but his scientific influence was much wider, through his lectures, his books, his scientific vision, and his extraordinary personality. Among the many official honors that he obtained, here we only want to mention that in 2006 he received an honorary doctorate from the Vietnam Academy of Science and Technology in Hanoi.

For a detailed obituary, we refer to [9].

This special issue comprises the following 9 papers (sorted by submission time) on various areas of mathematics and mathematical physics, whose authors had close ties to Eberhard Zeidler.

- Robert Finn: Capillary Graph-Interfaces "in-the-Large"

- Daniela D’Ascanio, Peter Gilkey, and Pablo Pisani: The Geometry of Locally Symmetric Affine Surfaces

- Wolfgang Hackbusch: Numerical Tensor Techniques for Multidimensional Convolution Products

- Felix Finster: Positive Functionals Induced by Minimizers of Causal Variational Principles

- Jürgen Jost, Enno Keßler, Jürgen Tolksdorf, Ruijun Wu, and Miaomiao Zhu: From Harmonic Maps to the Nonlinear Supersymmetric Sigma Model of Quantum Field Theory. At the Interface of Theoretical Physics, Riemannian Geometry and Nonlinear Analysis

- Kishore Marathe: From Moonshine to Mock Moonshine 
- Stefan Müller and Florian Schweiger: Estimates for the Green's Function of the Discrete Bilaplacian in Dimensions 2 and 3

- Harald Grosse and Raimar Wulkenhaar: How Prof. Zeidler Supported our Research on: Exact Solution of Quantum Field Theory Toy Models

- Christian Fleischhack: Spectra of Abelian $C^{*}$-Subalgebra Sums

We would like to thank all authors for their interesting contributions and all reviewers for their helpful comments.

And in turn, we, also in the name of the authors of this issue, would like to express our great gratitude for the support and scientific and human inspiration that Eberhard Zeidler has provided. We shall always remember his scientific vision of the unity of mathematics and physics and his great human personality.

Publisher's Note Springer Nature remains neutral with regard to jurisdictional claims in published maps and institutional affiliations.

\section{References}

1. Zeidler, E.: Nonlinear Functional Analysis and its Applications. I. Fixed-Point Theorems. Springer, New York (1986)

2. Zeidler, E.: Nonlinear Functional Analysis and its Applications. II/A. Linear Monotone Operators. Springer, New York (1990)

3. Zeidler, E.: Nonlinear Functional Analysis and its Applications. II/B. Nonlinear Monotone Operators. Springer, New York (1990)

4. Zeidler, E.: Nonlinear Functional Analysis and its Applications. III. Variational Methods and Optimization. Springer, New York (1985)

5. Zeidler, E.: Nonlinear Functional Analysis and its Applications. IV. Applications to Mathematical Physics. Springer, New York (1988)

6. Zeidler, E.: Quantum Field Theory. I. Basics in Mathematics and Physics. A Bridge Between Mathematicians and Physicists, 2nd edn. Springer, Berlin (2009)

7. Zeidler, E.: Quantum Field Theory. II. Quantum Electrodynamics. A Bridge Between Mathematicians and Physicists. Springer, Berlin (2009)

8. Zeidler, E.: Quantum Field Theory. III. Gauge Theory. A Bridge Between Mathematicians and Physicists. Springer, Berlin (2011)

9. Jost, J.: Eberhard Zeidler 1940-2016. Jahresbericht der Deutschen Mathematiker-Vereinigung 120(3), 221-228 (2018) 